\title{
Über die Einwirkung anorganischer Metallsäuren auf organische Säuren.
}

\author{
Von \\ ARTHUR ROSENHETM. \\ IV. Mitteilung.
}

Experimentell bearbeitet von Max Pratsch.

Am Schlusse der vorigen Mitteilung ${ }^{1}$ war gezeigt worden, in welcher Weise die Entstehung komplexer Anionen innerhalb eines Mischsalzes mit der Affinitätsgrörse der Bestandteile zusammen hängt. Wurden diese Affinitätsgröfsen mit den Symbolen $m s_{1}$ und $s_{2}$ bezeichnet, so mufste sich der Quotient $\frac{m-s_{2}}{s_{1}-s_{2}}$ für den Fall, dals ein Doppelsalz vorliege, der Zahl Eins oder Unendlich nähern, während er bei komplexsauren Salzen Werte mittlerer Grölse annahm.

Es war ferner darauf hingewiesen worden, dals dieser Quotient die Thatsache zum Ausdruck bringt, dals die „freien komplexen Säuren" zu vielen Reihen komplexsaurer Salze nicht existieren, sobald das Anion dieser Körper ein wenig beständiger Komplex ist, und dafs, wenn man hieraus die weiteren Folgerungen zieht, auch die Verbindungen mancher unbeständiger, komplexer Anionen mit schwach elektropositiven Kationen nicht darstellbar sein können. Denn wird in obigem Quotienten, besonders für die Fälle, in denen der Nenner ziemlich grofs ist, der Zähler durch Einsetzung eines niedrigen Wertes für $m$ kleiner, so nähert sich der Wert des Bruches Eins, ein Zeichen dafür, dafs das aus den betreffenden Bestandteilen gebildete Milchsalz ein Doppelsalz ist, und mithin wird dann Dissoziation innerhalb des sonst komplexen Anions stattfinden.

Dieser Einflufs wird sich naturgemäfs am deutlichsten bei den-

$1 Z$. anorg. Chem. 20, 281.

z. snorg. Chem, XXI. 
jenigen komplexsauren Verbindungen zeigen, deren komplexes Anion ziemlich unbeständig ist, d. h. in allen Fällen, wo die hypothetische, frei komplexe Säure eine sehr geringe Avidität haben würde. Es liegt, hier ein vollständiger Parallelismus mit den einfachen Säuren und Salzen vor, denn bekanntlich sind schwache Säuren, wie Kohlensäure, schweflige Säure etc., deren Hydrate in freiem Zustand auch nicht darstellbar sind, zur Salzbildung mit schwachen Basen, wie Thonerde, Chromoxyd u. s. w. in wässeriger Lösung nicht befähigt; es tritt entweder eine Salzbildung überhaupt nicht ein, oder es entstehen stark basische Salze.

Als ein Beweis dafür, dal's thatsächlich derartige Gesetzmälsigkeiten existieren, kann es angesehen werden, dals, während in der Litteratur die Salze starker, komplexer Säuren mit der Kieselwolframsäure, Phosphorwolframsäure etc. mit allen Metallbasen auf das genaueste beschrieben sind, zuverlässige Angaben über Versuche, dieselben bei schwach komplexen Säuren darzustellen, fast vollständig fehlen. Die negativen Ergebnisse solcher Versuche verursachen wahrscheinlich diese Lücken, deren Ausfüllung nach den obigen Ausführungen durchaus wünschenswert erscheint. Es war festzustellen, inwieweit in einem Mischsalze die Komplexität des Anions von der wechselnden Affinitätsgrörse des in das Salz einzuführenden Kations abhängig ist.

$\mathrm{Zu}$ diesem Zwecke führte Herr Max Platsch die in folgendem beschriebenen Versuche aus, indem er die Umsetzungen der in der II. Mitteilung ${ }^{1}$ beschriebenen aluminium-, chrom- und eisenoxalsauren Alkalien mit Metallchloriden studierte. Diese Versuche, durch die festgestellt werden sollte, wie stark die Affinitätsgröfse des Kations abgeschwächt werden kann, bis eine Zersetzung des komplexen Anions eintritt, indem der Reaktionsverlauf der doppelten Umsetzung studiert wurde, beanspruchen, wie gleich hier hervorgehoben werden mag, in Bezug auf die angeführten Gesetzmälsigkeiten nur gewissermafsen einen qualitativen Wert. Denn einerseits sind die Unterlagen für absolute Affinitätswerte, worauf am Schlusse dèr vorigen Mitteilung schou hingewiesen ist, noch nicht vorhanden, dann spielen aber auch bei doppelten Umsetzungen Faktoren, wie Löslichkeitsbeeinflussungen und Massenwirkungen, eine grolse Rolle, die hier bei den verschiedenen, sehr komplizierten Systemen nicht be،ücksichtigt werden konnten.

' ¿. anorg. Chem. 11, 175. 


\title{
Die Umsetzung von Thonerde-Chromoxyd und Eisenoxalaten mit Metallchloriden.
}

\author{
Von Max Platsch. ${ }^{1}$
}

\section{Umsetzungen der Aluminiumoxalate.}

Von den verschiedenen Reihen der aluminiumoxalsauren Salze ist die der Zusammensetzung $\mathrm{Al} \equiv(\mathrm{COO}-\mathrm{COOR})_{3}$ die beständigste und enthält, wie früher nachgewiesen wurde, nur unzweifelhaft komplexsaure Alkalisalze. Die vorliegenden Versuche wurde mit dem Ammoniumsalze dieser Reihe,

$$
\mathrm{Al}=\left(\mathrm{COO}-\mathrm{COO} . \mathrm{NH}_{4}\right)_{3}+{ }^{1}{ }_{2} \mathrm{H}_{2} \mathrm{O},
$$

ausgeführt. Dasselbe wurde in grofsen Mengen nach den früher gegebenen Vorschriften dargestellt und durch mehrfaches Umkrystallisieren gereinigt.

Bei den Versuchen wurden gewogene Mengen dieses Salzes in möglichst konzentrierten kalten Lösungen - in einigen besonders angegebenen Fällen wurde in der Hitze gearbeitet - mit Lösungen äquimolekularer Mengen der angewandten Metallchloride versetzt. Ausgefallene unlösliche Niederschläge wurden nach dem Klären der darüberstehenden Lösung abfiltriert, ausgewaschen und untersucht. Die Mutterlaugen wurden im Exsiccator über Schwefelsäure eingeengt und ca. 3-4 Anschüsse analysiert.

\section{Umsetzung mit Baryumchlorid.}

Bei sofortigem Zusatz der ganzen Baryumchloridmenge zu der Lösung des Thonerdeoxalats fiel eine grofse Menge Baryumoxalat aus; wurde jedoch das Baryumchlorid in kleinen Portionen unter gründlichem Umrühren eingetragen, so blieb die Ausscheidung aufserordentlich gering. Der entstandene Niederschlag wurde schnell abfiltriert und qualitativ nachgewiesen, dals nur reines, thonerdefreies Baryumoxalat vorlag. Das klare Filtrat erstarrte nach kurzem Stehen zu einem Gemisch seidenglänzender, weifser Nadeln. Wie wiederholte Analysen und die mikroskopische Besichtigung zeigten, waren dieselben jedoch stets mit geringen Mengen von Baryumoxalat verunreinigt. Das Salz wurde deswegen trotz seiner Schwerlöslichkeit aus kaltem Wasser unter vorsichtigem Eindampfen bei gelinder Hitze - beim Umkrystallisieren in der Siedehitze trat stets eine

\footnotetext{
1 Inaug.-Dissert. (Basel 1898), S. 5-41.
} 
starke Zersetzung und bedeutende Abscheidung von Baryumoxalat ein - umkrystallisiert und so schliefslich in ganz analysenreinem Zustand gewonnen.

Das Salz erwies sich als ein Baryumammoniumdoppelsalz der Zusammensetzung

\begin{tabular}{|c|c|c|c|c|}
\hline \multirow{7}{*}{$\begin{array}{l}2 \mathrm{BaO} \\
\left(\mathrm{NH}_{4}\right)_{2} \mathrm{O} \\
\mathrm{Al}_{2} \mathrm{O}_{6} \\
6 \mathrm{C}_{2} \mathrm{O}_{8} \\
4 \mathrm{H}_{2} \mathrm{O}\end{array}$} & \multicolumn{4}{|c|}{$\mathrm{Al} \equiv\left(\mathrm{C}_{2} \mathrm{O}_{4}\right)_{3} \mathrm{BaNH}_{4}+2 \mathrm{H}_{2} \mathrm{O}$} \\
\hline & & Berechnet: & Erh & alten: \\
\hline & $=306$ & $31.74 \%$ & 31.52 & $31.54 \%$ \\
\hline & $=52$ & 5.39 & 5.29 & 5.18, \\
\hline & $=102$ & 10.58, & 10.63 & $10.73 "$ \\
\hline & $=432$ & 44.82 & 44.52 & $44.81 "$ \\
\hline & $=72$ & $7.47 \%$ & & \\
\hline & 964 & $100.00 \%$ & & \\
\hline
\end{tabular}

Sämtliche spätere Anschüsse, sowie mehrfache Wiederholungen des Versuches ergaben dasselbe Doppelsalz. Bei Anwendung von Kaliumthonerdeoxalat wurde ein entsprechendes Baryumkaliumdoppelsalz erhalten.

Diese Verbindungen sind, wie ihr Verhalten beim Umkrystallisieren, sowie bei der Darstellung zeigt, in Bezug auf das komplexe Anion schon wesentlich unbeständiger, als die Alkalithonerdeoxalate. Bei der Behandlung mit warmem Wasser tritt hier sehr schnell Zersetzung in Baryumoxalat und oxalsaure Thonerde ein, während die Alkalithonerdeoxalate sich quantitativ ohne die geringste Zersetzung aus siedender Lösung umkrystallisieren lassen.

In der II. Mitteilung ${ }^{1}$ war durch Einwirkung von überschüssigem Chlorbaryum auf eine Lösung von oxalsaurer Thonerde ein einfaches Baryumsalz, $\left[\mathrm{Al}\left(\mathrm{C}_{2} \mathrm{O}_{4}\right)_{3}\right]_{2} \mathrm{Ba}_{3}+6 \mathrm{H}_{2} \mathrm{O}$, erhalten worden, das ebenfalls grofse Zersetzlichkeit in wässeriger Lösung zeigte. ${ }^{2}$

\section{Umsetzung mit Strontiumchlorid.}

Die Reaktion verliert, wie beim Baryumsalze, nur dafs wesentlich gröfsere Mengen Strontiumoxalat sich ausschieden. Ebenso war die Reinigung des Salzes entsprechend schwieriger, und erst durch 3-4 maliges Umkrystallisieren war ein einigermafsen analysenreines Produkt zu erhalten.

${ }^{1}$ Z. anorg. Ohem. 11, 180.

${ }^{2}$ Rees Reece (Compt. rend. 21, 1116) beschreibt dasselbe Salz mit $10 \mathrm{H}_{2} \mathrm{O}$. 
Während der erste Anschuls also, wie qualitativ nachgewiesen wurde, Strontiumoxalat enthielt, ergaben die späteren Anschüsse ein komplexes Strontiumammoniumsalz der Zusammensetzung:

$$
\begin{aligned}
& \mathrm{Al} \equiv\left(\mathrm{C}_{2} \mathrm{O}_{4}\right)_{3} \mathrm{SrNH}_{4}+5 \mathrm{H}_{2} \mathrm{O} \text {. }
\end{aligned}
$$

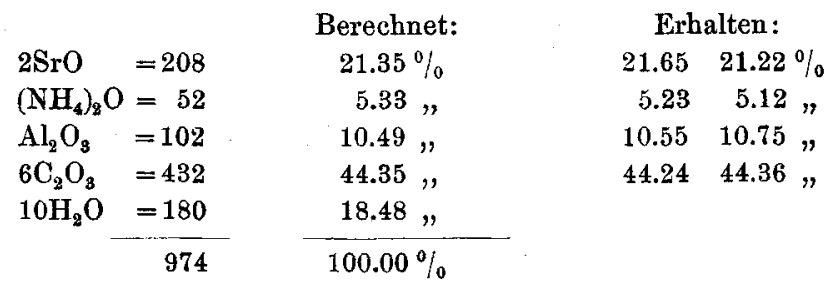

In diesem Strontiumdoppelsalze ist das komplexe Anion noch wesentlich unbeständiger, wie in dem Baryumsalze. ${ }^{1}$

3. Umsetzungen mit Calcium-Magnesium- und Zinkchlorid.

Bei der Behandlung des Ammoniumthonerdeoxalates mit äquivalenten Mengen einer Calciumchloridlösung konnte ein, den vorigen Körpern entsprechendes Calciumsalz nicht erhalten werden. Aus der anfangs klaren Lösung schieden sich nach und nach, sowohl beim freiwilligen Verdunsten über Schwefelsäure, wie beim Konzentrieren der Lösung bei gelinder Temperatur, grofse Mengen von Calciumoxalat $a b$, denen später Krystalle von Ammoniumchlorid beigemengt waren. Es hinterblieb schliefslich eine syrupöse Lauge, die fast nur Thonerde und Oxalsäure, sowie geringe Mengen von Chlorammonium enthielt. Sie stellte das schon früher beschriebene, nicht krystallisierende Thonerdeoxalat dar.

Auch REECE ${ }^{2}$ hatte ein Calciumsalz auf demselben Wege wie das Baryum- und Strontiumsalz nicht erhalten können.

Um zu untersuchen, ob vielleicht auf anderem Wege ein Calciumsalz darstellbar sei, wurde eine Lösung der hypothetischen Thonerdeoxalsäure $\mathrm{Al} \equiv(\mathrm{COO}-\mathrm{COOH})_{3}$ durch Absättigung gewogener Mengen Oxalsäure mit Aluminiumoxydhydrat und Zusatz der gleichen Mengen Oxalsäure dargestellt. Diese Lösung wurde mit äquivalenten Mengen von Calciumkarbonat bei gelinder Temperatur behandelt, doch trat auch hierbei vollständige Zersetzung unter allmählicher Abscheidung von Calciumoxalat ein, und es hinterblieb nur der oben beschriebene Syrup.

1 Rees Reece (l. c.) beschreibt ein Salz $3 \mathrm{SrO} . \mathrm{Al}_{2} \mathrm{O}_{3} \cdot 6 \mathrm{C}_{2} \mathrm{O}_{3}+18 \mathrm{H}_{2} \mathrm{O}$.

2 l. e. 
Ganz entsprechende Erscheinungen wurden beobachtet, als Lösungen des Ammoniumthonerdeoxalats mit äquivalenten Mengen der Chloride von Magnesium und Zink versetzt wurden. Ebenso trat vollständiger Zerfall des komplexen Anions bei Umsetzungen mit Eisenchlorür und Eisenchlorid ein. Im letzteren Falle ging der Zersetzung eine Reduktion des Eisenchlorids voraus.

Diese Beobachtungen schliefsen sich ganz gesetzmälsig den vorigen Thatsachen an. Während die Affinitätsgrölse von Baryum und Strontium noch gerade hinreicht, um mit der Aluminiumoxalsäure, wenn auch unbeständige Salze zu bilden, ist die der in der Spannungsreihe folgenden Elemente, Magnesium, Zink und Eisen, nicht mehr grofs genug dazu.

Bei den Umsetzungen mit den Chloriden der in der Spannungsreihe nunmehr folgenden Elemente tritt ein neuer, für den Reaktionsverlauf wichtiger Faktor hinzu: die Salze der schwachen Metallbasen mit starken Säuren, soweit sie wasserlöslich sind, zeigen saure Reaktion.

\section{Umsetzung mit Kobaltchlorid.}

Hierbei schied sich alsbald ein blafsroter, krystallinischer Niederschlag aus, der, wie die qualitative und quantitative Untersuchung erwies, reines Kobaltoxalat war. Der zweite Anschufs brachte aus einer über Schwefelsäure stark eingeengten Mutterlauge ein Gemisch roter monokliner Nadeln des bekannten Doppelsalzes $\mathrm{NH}_{4} \mathrm{CoCl}_{3}+6 \mathrm{H}_{2} \mathrm{O}$ und reinen Chlorammoniums.

Aus der Mutterlange dieses Anschusses, die, wie die Farblosigkeit der Lösung zeigte, vollständig frei von Kobalt war, schieden sich bei weiterem Einengen über Schwefelsäure nunmehr mikrokrystallinische, farblose Niederschläge ab, die grofse Mengen von Aluminium, Oxalsäure und Ammoniak und geringe Mengen von Chlor enthielten.

Die Analysen der dritten und vierten Krystallisation führten zu den folgenden Werten: ${ }^{1}$

$\begin{array}{rc}\text { 3. Anschufs: } & \text { 4. Anschuls: } \\ 7.44 \% \mathrm{NH}_{3} . & 6.83 \% \mathrm{NH}_{3} . \\ 2.41 " \mathrm{Cl} & 1.83 \% \mathrm{Cl} . \\ 20.92 " \mathrm{Al}_{2} \mathrm{O}_{3} . & 18.65 " \mathrm{Al}_{2} \mathrm{O}_{3} . \\ 51.64 " \mathrm{C}_{2} \mathrm{O}_{3} . & 51.19 " \mathrm{C}_{2} \mathrm{O}_{3} .\end{array}$

1 Die angegebenen Werte sind stets das Mittel aus mehreren gut unter einander stimmenden Analysen. 
Das Chlor kann in diesem Gemisch nur als Chlorammonium gebunden sein. Zieht man daher die dem Chlor entsprechende Ammoniakmenge von dem gefundenen Ammoniakgehalt ab, so kommt man für den dritten Anschufs zu fọlgenden Werten:

\begin{tabular}{c|l|c}
\hline $\begin{array}{c}\text { Molekularquotient } \\
\text { gefunden }\end{array}$ & \multicolumn{1}{c|}{$\begin{array}{c}\text { Molekularquotient } \\
\text { reduziert }\end{array}$} & \multicolumn{1}{c}{$\begin{array}{c}\text { Prozente } \\
\text { reduziert }\end{array}$} \\
\hline \hline $\mathrm{NH}_{3}=0.44$ & $0.37=6.29 \% \mathrm{NH}_{3}=$ & $9.62\left(\mathrm{NH}_{4}\right)_{2} \mathrm{O}$ \\
$\mathrm{Al}_{2} \mathrm{O}_{3}=0.21$ & 0.21 & $20.92 \mathrm{Al}_{2} \mathrm{O}_{3}$ \\
$\mathrm{C}_{2} \mathrm{O}_{3}=0.72$ & 0.72 & $51.64 \mathrm{C}_{2} \mathrm{O}_{3}$ \\
$\mathrm{Cl}=0.07$ & & \\
\hline
\end{tabular}

$82.18 \%$.

Die an das Chlor gebundene Ammoniakmenge ist aus den gefundenen Molekularquotienten berechnet; aus den reduzierten Molekularquotienten wurde alsdann die verbleibende Ammoniakmenge zurückgerechnet. Berechnet man nun die gefundenen reduzierten Zahlen - der Wassergehalt dieser stets stark wasserhaltigen Gemische wurde niemals direkt bestimmt - auf wasserfreie Substanz, so ergeben sich folgende Werte:

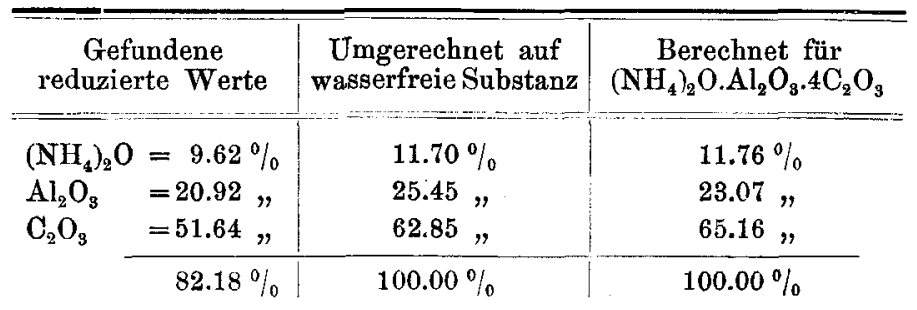

Dieselbe Rechnung, für den vierten Anschufs ausgeführt, ergiebt:

\begin{tabular}{|c|c|c|c|c|}
\hline $\begin{array}{l}\text { Molekularquot. } \\
\text { gefunden }\end{array}$ & $\begin{array}{l}\text { Molekularquot. } \\
\text { reduziert }\end{array}$ & Prozente & $\begin{array}{l}\text { Umgerechnet } \\
\text { auf wasser- } \\
\text { freieSubstanz }\end{array}$ & $\begin{array}{l}\text { Berechnet für } \\
\left(\mathrm{NH}_{4}\right)_{2} \mathrm{O} . \\
\mathrm{Al}_{2} \mathrm{O}_{3} .4 \mathrm{C}_{2} \mathrm{O}_{3}\end{array}$ \\
\hline \multirow{5}{*}{$\begin{array}{l}\mathrm{NH}_{3}=0.40 \\
\mathrm{Cl}=0.05 \\
\mathrm{Al}_{3} \mathrm{O}_{3}=0.18 \\
\mathrm{C}_{2} \mathrm{O}_{3}=0.71\end{array}$} & \multirow{4}{*}{$\begin{array}{l}0.35=5.95 \% \mathrm{NH}_{8}= \\
0.18= \\
0.71=\end{array}$} & $9.10\left(\mathrm{NH}_{4}\right)_{2} \mathrm{O}$ & $11.52 \%$ & \multirow{5}{*}{$\begin{array}{l}11.76 \% \\
-\overline{2} \% \\
65.07 \%\end{array}$} \\
\hline & & - & - & \\
\hline & & $18.65 \mathrm{Al}_{2} \mathrm{O}_{3}$ & 23.62, & \\
\hline & & $51.19 \mathrm{C}_{2} \mathrm{O}_{8}$ & $64.84 "$ & \\
\hline & & 78.94 & $100.00 \%$ & \\
\hline
\end{tabular}

Aus diesen Werten, die in anbetracht der Schwierigkeiten, mit denen die Untersuchungen solcher Mischungen verknüpft sind, 
gut übereinstimmen, geht klar hervor, dafs durch die Einwirkung von Kobaltchlorid auf das Ammoniumthonerdeoxalat

$$
\text { die Verbindung } \begin{gathered}
\mathrm{Cl}=\left(\mathrm{COO}-\mathrm{COO} \cdot \mathrm{NH}_{4}\right)_{3}+2^{1}{ }_{2} \mathrm{H}_{2} \mathrm{O} \\
\mathrm{COO}
\end{gathered}>\mathrm{Al}-\mathrm{COO} . \mathrm{COONH}_{4}+2^{1}{ }_{2} \mathrm{H}_{2} \mathrm{O}^{1} \text { entstanden ist. }
$$

Die Reaktion verläuft im wesentlichen nach folgender Gleichung:

$$
\begin{gathered}
2 \mathrm{Al} \equiv\left(\mathrm{COO}-\mathrm{COO} . \mathrm{NH}_{4}\right)_{3}+3 \mathrm{CoCl}_{2}=2 \mathrm{Al}\left(\mathrm{C}_{2} \mathrm{O}_{4}\right) \mathrm{COO}-\mathrm{COONH}_{4} \\
+\mathrm{CoC}_{2} \mathrm{O}_{4}+\mathrm{CoNH}_{4} \mathrm{Cl}_{3}+3 \mathrm{NH}_{4} \mathrm{Cl} .
\end{gathered}
$$

5. Umsetzungen mit Nickel-Cadmium und Kupferchlorid.

Der Verlauf der Reaktion war hierbei genau derselbe wie im vorigen Falle; erst Ausscheidung des Metalloxalats, dann Krystallisation von Chlorammonium und von überschüssigem Metallchlorid bezw. eines Doppelchlorids wie $\mathrm{NH}_{4} \mathrm{CdCl}_{3}+{ }_{1}^{1}{ }_{2} \mathrm{H}_{2} \mathrm{O}$, und in den letzten Anschüssen Entstehung desselben Ammoniumthonerdeoxalats $\mathrm{Al}\left(\mathrm{C}_{2} \mathrm{O}_{4}\right) \mathrm{C}_{2} \mathrm{O}_{4} \mathrm{NH}_{4}$, vermischt mit geringen Mengen von Chlorammonium. Die Analysen dieser letzten Anschüsse ergaben sehr gut stimmende Werte, ${ }^{2}$ auf die hier nur verwiesen sei.

Da bei den übrigen in Betracht kommenden Metallen, wie Silber, Blei, Quecksilberoxydul, die Chloride schwer löslich sind, so wurden bei ihnen Umsetzungen mit Nitraten und Acetaten versucht, doch führten dieselben einerseits wegen unangenehmer Einwirkung der Salpetersäure auf die hinterbleibenden Laugen, andererseits wegen der Schwierigkeit, die geringen Beimengungen von Salpetersäure und Essigsäure in diesen Gemischen quantitativ genau zu bestimmen, zu keinen verwendbaren Resultaten.

\section{Umsetzungen der Chromoxalate.}

A. Einwirkung auf das blaue Ammoniumchromoxalat,

$$
\mathrm{Cr} \equiv\left(\mathrm{COO}-\mathrm{COO} . \mathrm{NH}_{4}\right)_{3}+3 \mathrm{H}_{2} \mathrm{O} \text {. }
$$

Diese Versuche, die in ganz derselben Weise ausgeführt wurden, wie die im vorigen Abschnitt beschriebenen, führten $\mathrm{zu}$ sehr ähn-

1 Vergl. Z. anorg. Chem. 11, 193.

- Vergl. Puatscr, lnaug.-Dissert. S. 17-22. Die dort bei der Umsetzung mit $\mathrm{CdCl}_{2}$ einmal angenommene Entstehung des Salzes $\mathrm{Al}{\left.\mathbb{\mathrm { COO }}-\mathrm{COO} \cdot \mathrm{NH}_{4}\right)_{2}}_{\text {ist }}^{\mathrm{OH}}$ auf ein kleines analytisches Versehen zurückzuführen. Es bildet sich stets $\mathbf{n}$ ur die oben angegebene Verbindung, und ist demgemäls diese Angabe zu korrigieren. 
lichen Ergebnissen, die im folgenden kurz zusammengestellt sein mögen.

1. Umsetzung mit Baryumchlorid.

Ausscheidung von Baryumoxalat in geringen Mengen und Krystallisation blaugrüner verfiltzter Nadeln. Die letzteren durch Omkrystallisieren aus kaltem Wasser gereinigt - in der Wärme tritt Zersetzung in Baryumoxalat ein - erwiesen sich als

$$
\left[\mathrm{Cr} \equiv(\mathrm{COO}-\mathrm{COO})_{3}\right]_{2} \mathrm{Ba}_{3}+14 \mathrm{H}_{2} \mathrm{O} .^{1}
$$

\begin{tabular}{|c|c|c|c|c|}
\hline \multirow[b]{2}{*}{$3 \mathrm{BaO}$} & \multirow[b]{2}{*}{$=459$} & \multirow{2}{*}{$\begin{array}{c}\text { Berechnet: } \\
35.41 \%\end{array}$} & \multicolumn{2}{|c|}{ Erhalten: } \\
\hline & & & 35.55 & $35.64 \%$ \\
\hline $\mathrm{Cr}_{2} \mathrm{O}_{3}$ & $=153$ & 11.80 & 11.92 & 11.73 \\
\hline $6 \mathrm{C}_{2} \mathrm{O}_{3}$ & $=432$ & $33.35 "$ & 33.27 & 33.56, \\
\hline $14 \mathrm{H}_{2} \mathrm{O}$ & $=252$ & 19.44 & & \\
\hline & 1296 & $100.00 \%$ & & \\
\hline
\end{tabular}

2. Umsetzung mit Strontiumchlorid.

Grofse Mengen von Strontiumoxalat und ein komplexes, in blaugrünen Nadeln krystallisierendes Salz, das wesentlich zersetzlicher ist, als die Baryumverbindung. Das durch wiederholtes Umkrystallisieren in der Kälte gereinigte Produkt hatte die Zusammensetzung:

\begin{tabular}{cccc} 
& $\mathrm{Cr}\left(\mathrm{C}_{2} \mathrm{O}_{4}\right)_{3} \cdot \mathrm{Sr} \cdot \mathrm{NH}_{4}+5 \mathrm{H}_{2} \mathrm{O}$. \\
& Berechnet: & \multicolumn{2}{c}{ Erhalten: } \\
$2 \mathrm{SrO}=208$ & $20.29 \%$ & 20.19 & $20.56 \%$ \\
$\left(\mathrm{NH}_{4}\right)_{2} \mathrm{O}=52$ & $5.07 \%$ & 5.21 & $5.04 \%$ \\
$\mathrm{Cr}_{2} \mathrm{O}_{2}=153$ & $14.93 \%$ & 14.32 & $14.55 \%$ \\
$6 \mathrm{C}_{2} \mathrm{O}_{3}=432$ & $42.15 \%$ & 41.71 & $41.54 \%$ \\
$10 \mathrm{H}_{2} \mathrm{O}=180$ & $17.56 \%$ & & \\
\hline & 1025 & $100.00 \%$ &
\end{tabular}

3. Umsetzungen mit Calcium-Magnesium und Zinkchlorid.

Bei gewöhnlicher Temperatur schied sich zuerst in grofsen Mengen das Metalloxalat aus, dann krystallisierte Chlorammonium sowie das Metallchlorid bisweilen getrennt, bisweilen zu Doppelsalzen vereinigt und es hinterblieb ein nicht krystallisierender Syrup von oxalsaurem Chromoxyd.

Wurde die Umsetzung des Chromammoniumoxalats mit Calciumchlorid bei $-15^{\circ}$ in einer Kältemischung ausgeführt, so gelang es,

1 Erhalten mit verschiedenem Wassergehalt von Reece (1. c.), E. A. Werner (Journ. Chem. Soc. 53, 406 und 609) und ClaRke und Kebler (Ber. deutsch. chem. Ges. 14, 36 und 1639). 
sehr kleine Mengen eines in Nadeln krystallisierenden Salzes zu erhalten, das, wie die qualitative Untersuchung zeigte, wahrscheinlich ein Doppelsalz ${ }^{1}$ der Zusammensetzung

$$
\mathrm{Cr}\left(\mathrm{C}_{2} \mathrm{O}_{4}\right)_{3} \mathrm{Ca}_{2} \mathrm{NH}_{4}
$$

war. Der Körper zersetzt sich beim Umkrystallisieren bei gewöhnlicher Temperatur sofort in Calciumoxalat, und es konnten nicht hinreichende Mengen zur quantitativen Untersuchung gewonnen werden.

4. Umsetzungen mit Kobalt-, Nickel- und Kupferchlorid.

Der Verlauf der Reaktion war auch hier genau derselbe, wie bei dem Ammoniumaluminiumoxalat: erst Ausscheidung des Metalloxalats, dann Krystallisation des Metallchlorids mit Chlorammonium zum Doppelsalz vereinigt und dann eines Gemisches des letzteren Salzes mit dem roten Ammoniumchromoxalate $\prod_{\mathrm{COO}}^{\mathrm{COO}} \mathrm{Cr}-\mathrm{COO}-\mathrm{COO} \cdot \mathrm{NH}_{4}$. Dieses konnte hier vermöge seiner hervorstechenden Farbe und seiner charakteristischen Eigenschaften ${ }^{2}$ schon qualitativ identifiziert werden.

\section{Umsetzung mit Cadmiumchlorid.}

Diese verlief in etwas abweichender Weise. Die erste Ausscheidung brachte bedeutende Mengen von neutralem Cadmiumoxalat, dann krystallisierte Cadmiumchloridchlorammonium $\mathrm{CdCl}_{2} \cdot \mathrm{NH}_{4} \mathrm{Cl}+$ $1 / 2$ aq. in charakteristischen Nadeln aus, der dritte Anschufs endlich brachte eine reiche Ausbeute tiefblauer, prismatischer Krystalle, gemengt mit Cadmiumchloridchlorammonium. Dieselben lieisen sich durch schnelles Behandeln mit kaltem Wasser fast vollständig von dem Cadmiumchloriddoppelsalze befreien. Ihre Analyse führte zu der Formel:

\begin{tabular}{|c|c|c|c|}
\hline \multirow{6}{*}{$\begin{array}{l}\left(\mathrm{NH}_{4}\right)_{2} \mathrm{O} \\
2 \mathrm{CdO} \\
\mathrm{Cr}_{2} \mathrm{O}_{3} \\
6 \mathrm{C}_{2} \mathrm{O}_{3} \\
20 \mathrm{H}_{2} \mathrm{O}\end{array}$} & \multicolumn{3}{|c|}{$\mathrm{Cr} \equiv\left(\mathrm{C}_{2} \mathrm{O}_{4}\right)_{3} \mathrm{Cd} \cdot \mathrm{NH}_{4}+10 \mathrm{H}_{2} \mathrm{O}}$. \\
\hline & $=52$ & Berechnet: & $\begin{array}{c}\text { Erhalten: } \\
\quad \mathbf{4 . 1 6} \%\end{array}$ \\
\hline & $=256$ & $20.45 \#$ & 20.48 \\
\hline & $=152$ & 12.14, & $12.88 "$ \\
\hline & $=432$ & $34.50 "$ & 34.56, \\
\hline & $=380$ & 28.76, & \\
\hline & 1252 & $100.00 \%$ & \\
\hline
\end{tabular}

1 E. A. Werner (l. c.) beschreibt ein analoges Salz $\operatorname{Cr}\left(\mathrm{C}_{2} \mathrm{O}_{4}\right)_{3} \mathrm{CaK}$.

${ }^{2}$ Vergl. II. Mitteilung (Z. anorg. Chem. 11, 165). 
B. Einwirkung auf das rote Kaliumchromoxalat,

$$
\mathrm{C}_{2} \mathrm{O}_{4}=\mathrm{Cr}-\mathrm{C}_{2} \mathrm{O}_{4} \mathrm{~K}+5 \mathrm{H}_{2} \mathrm{O} \text {. }
$$

Um die Bedingungen näher zu untersuchen, unter denen der oben mehrfach beobachtete Übergang der komplexen Oxalate der Zusammensetzung $\stackrel{\mathrm{II}}{\mathrm{M}} \equiv(\mathrm{COO}-\mathrm{COOR})_{3}$ in die der Zusammensetzung $\mathrm{C}_{2} \mathrm{O}_{4}=$ MII.COO-COOR stattfindet, wurden die Umsetzungen des "roten" Kaliumchromoxalats, das nach den in der II. Mitteilung ${ }^{1}$ gemachten Angaben leicht in grofser Menge darstellbar ist, ausgeführt. Da, wie in der zitierten Stelle näher begründet ist, die wässerigen Lösungen der "roten" Salze in der Hitze wahrscheinlich ein anders konstituiertes Anion enthalten, als in der Kälte, indem unter Aufnahme von Wasser ein Übergang von $\mathrm{C}_{2} \mathrm{O}_{4}=\mathrm{Cr}-\mathrm{C}_{2} \mathrm{O}_{4} \mathrm{~K}$ in $\mathrm{Cr} / \mathrm{C}_{2} \mathrm{O}_{4} \mathrm{H}$ stattfindet, so wurden stets Parallelversuche ausgeführt und einmal die Umsetzung und Krystallisation in der Wärme bei ungefähr $50-60^{\circ}$ auf dem Wasserbade, dann bei gewöhnlicher Temperatur im Exsiccator über Schwefelsäure beobachtet. Es möge gleich hier hervorgehoben werden, dals stets beide Versuche zu denselben Ergebnissen führten.

1. Umsetzung mit Baryumchlorid.

Die ersten Anschüsse brachten reiche Ausbeuten blaugrüner Krystallnadeln. Zuletzt verblieb ein nicht krystallisierender Syrup, der frei von Oxalsäure war. Die blaugrünen Nadeln erwiesen sich als ein Kaliumbaryumdoppelsalz der Formel:

$$
\mathrm{Cr} \equiv\left(\mathrm{C}_{2} \mathrm{O}_{4}\right)_{3} \mathrm{BaK}+2 \mathrm{H}_{2} \mathrm{O} \text {. }
$$

Berechnet:

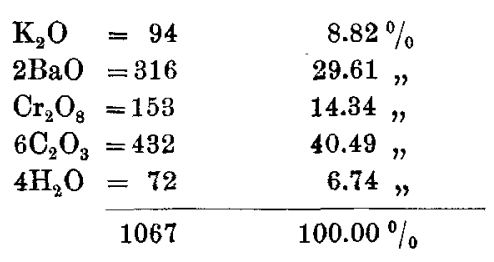

Erhalten:

I.

$8.89 \% \quad 8.96 \%$

$29.07 \% \quad 29.51 \quad 28.38 \%$

$14.62 " \quad 13.66 \quad 14.70$,

40.54,

Unter I sind die in der Wärme, unter II die bei gewöhnlicher Temperatur erhaltenen Krystallisationen untersucht.

1 Z. anorg. Chem. 11, 209. 
Nach diesem Ergebnisse vollzieht sich die Umsetzung wahrscheinlich nach der Gleichung:

$$
3 \mathrm{C}_{2} \mathrm{O}_{4}=\mathrm{Cr}-\mathrm{C}_{2} \mathrm{O}_{4} \mathrm{~K}+2 \mathrm{BaCl}_{2}=2 \mathrm{Cr}\left(\mathrm{C}_{2} \mathrm{O}_{4}\right)_{3} \mathrm{BaK}+\mathrm{CrCl}_{3}+\mathrm{KCl} \text {. }
$$

2. Umsetzung mit Strontiumchlorid.

Dieselbe ergab ganz das entsprechende Resultat. Das Strontiumkaliumdoppelsalz

$$
\mathrm{Cr}\left(\mathrm{C}_{2} \mathrm{O}_{4}\right)_{3} \mathrm{SrK}+4 \mathrm{H}_{2} \mathrm{O}
$$

krystallisiert in blauen, wohlausgebildeten Nadeln.

\begin{tabular}{|c|c|c|}
\hline $\mathrm{K}_{2} \mathrm{O}$ & $=94$ & $9.13 \%$ \\
\hline $2 \mathrm{SrO}$ & $=208$ & $20.17 "$ \\
\hline $\mathrm{Cr}_{2} \mathrm{O}_{3}$ & $=153$ & 14.84, \\
\hline $6 \mathrm{C}_{2} \mathrm{O}_{3}$ & $=432$ & $41.90 "$ \\
\hline $8 \mathrm{H}_{2} \mathrm{O}$ & $=144$ & 13.96 \\
\hline & 1031 & $100.00 \%$ \\
\hline
\end{tabular}

Berechnet:
Erhalten:

I.

$10.84 \%$

18.75,

14.34,

42.19,
II.

$18.16 \%$

15.46,

Umsetzung mit äquivalenten Mengen von Calcium- oder Magnesiumchlorid oder mit den Chloriden von Metallen wie Kupfer und Cadmium führten zu keinen krystallisierenden Produkten. Beim langen Stehen im Exsiccator schieden sich die beiden Bestandteile unverändert wieder aus. Ebensowenig wurden die Resultate verändert, als statt äquivalentér Mengen der Metallchloride Überschüsse, und zwar zwei oder drei Moleküle derselben angewendet wurden. Baryum- und Strontiumchlorid ergaben die obigen Doppelsalze, die anderen Chloride führten keine Umsetzung herbei.

\section{Umsetzungen der Eisenoxalate.}

Als Ausgangsmaterial wurde auch hier das Ammoniumsalz, die bekannte Verbindung $\mathrm{Fe} \equiv\left(\mathrm{COO}-\mathrm{COO} . \mathrm{NH}_{4}\right)_{3}+3 \mathrm{H}_{2} \mathrm{O}$, verwendet.

\section{Umsetzung mit Baryumchlorid.}

Hierbei schieden sich erst ganz ebenso, wie bei den entsprechenden Versuchen mit Thonerde- und Chromoxalaten, bedeutende Mengen von Baryumoxalat aus, dann krystallisierte eine grofse Ausbeute grüngelber Nadeln, die erst durch wiederholte Umkrystallisation aus kalter Lösung analysenrein erhalten wurden. 


\begin{tabular}{|c|c|c|c|}
\hline \multicolumn{4}{|c|}{$\left[\mathrm{Fe}\left(\mathrm{C}_{2} \mathrm{O}_{4}\right)_{3}\right]_{2} \mathrm{Ba}_{3}+22 \mathrm{H}_{2} \mathrm{O}$} \\
\hline $3 \mathrm{BaO}$ & $=316$ & $\begin{array}{c}\text { Berechnet: } \\
31.72 \%\end{array}$ & $\begin{array}{c}\text { Erhalten: } \\
31.86 \%\end{array}$ \\
\hline $\mathrm{Fe}_{2} \mathrm{O}_{3}$ & $=160$ & $11.05 \%$ & 10.95, \\
\hline $6 \mathrm{C}_{2} \mathrm{O}_{3}$ & $=432$ & 29.87 & 30.08, \\
\hline $22 \mathrm{H}_{2} \mathrm{O}$ & $=396$ & 27.36, & \\
\hline & 1304 & $100.00 \%$ & \\
\hline
\end{tabular}

2. Umsetzung mit Strontiumchlorid.

Neben Ausscheidung grofser Mengen von Strontiumoxalat, Krystallisation eines ebenfalls in gelbgrünen Nadeln krystallisierenden Strontiumammoniumferrioxalats.

\begin{tabular}{lcrr} 
& $\mathrm{Fe}\left(\mathrm{C}_{2} \mathrm{O}_{4}\right)_{3} \mathrm{SrNH}_{4}+6 \mathrm{H}_{2} \mathrm{O}$. \\
Berechnet: & \multicolumn{2}{c}{ Erhalten: } \\
$2 \mathrm{SrO}=208$ & $19.40 \%$ & 19.22 & $19.15 \%$ \\
$\left.\mathrm{NH}_{4}\right)_{2} \mathrm{O}=52$ & $4.89 "$ & 4.90 & $4.57 "$ \\
$\mathrm{Fe}_{2} \mathrm{O}_{3}=160$ & $15.05 "$ & 14.99 & $15.14 "$ \\
$6 \mathrm{C}_{3} \mathrm{O}_{8}=432$ & $40.44 "$ & 40.63 & $40.56 "$ \\
$12 \mathrm{H}_{2} \mathrm{O}=216$ & $20.22 "$ & & \\
\hline 1068 & $100.00 \%$ &
\end{tabular}

3. Umsetzung mit Calciumchlorid.

Hierbei schieden sich grofse Mengen von Calciumoxalat aus, und die Lösung nahm die tiefgelbe Färbung von Eisenchlorid an. Der zweite Anschuls brachte eine bedeutende Ausscheidung prismatischer, schwach gelbgefärbter Nadeln, schliefslich hinterblieb ein nicht krystallisierender, tiefgelber Syrup, der neben zahlreichen Zersetzungsprodukten Eisenoxyd und das bekannte gelbe Eisenoxyduloxalat ergab. Die Nadeln erwiesen sich bei wiederholten Analysen als das bekannte Doppelsalz ${ }^{1} \quad \mathrm{CaC}_{2} \mathrm{O}_{4}+\mathrm{CaCl}_{2}+7 \mathrm{H}_{2} \mathrm{O}$. Es war also hier eine vollständige Zersetzung des komplexen Eisenoxydoxalats eingetreten.

Dasselbe geschah bei der Umsetzung mit allen anderen $\mathrm{Me}$ tallchloriden. Sowohl Magnesium- und Zinkchlorid, wie Kobalt-, Cadmium- und Kupferchlorid veranlafsten die vollständige Ausscheidung des entsprechenden neutralen unlöslichen Metalloxalats, die Bildung von Eisenchlorid und die schlielsliche Krystallisation des bekannten Eisenoxyduloxalats und von Chlorammonium.

1 Fritsche, Pogg. 'Ann. 28, 121. 
IV.

Ein Überblick über die bei obigen Versuchen erhaltenen Resultate zeigt, dafs thatsächlich die komplexen Anionen der Aluminium-, Chrom- und Eisenalkalioxalate mit schwach elektropositiven Kationen Salze nicht bilden.

Die Baryum- und Strontiumsalze dieser komplexen Säuren sind noch bei gewöhnlicher Temperatur existenzfähig, wenn auch die Baryumsalze ziemlich und in wesentlich grölserem Mal'se die Strontiumsalze zersetzlich sind, wie ihr Zerfall in Erdoxalate und Aluminium-, bezw. Chrom- oder Eisenoxalat beim Umkrystallisieren beweist. Die Calciumsalze dagegen bilden sich bei gewöhnlicher Temperatur überhaupt nicht und nur ein aufserordentlich unbeständiges Calciumchromoxalat konnte bei sehr niedriger Temperatur erhalten werden.

Die nunmehr in der elektrochemischer Spannungsreihe folgenden Elemente, Magnesium und Zink, bilden keine Salze mit den komplexen Anionen. Bei der doppelten Umsetzung tritt ein vollständiger Zerfall des Komplexes ein. Abweichend verhält sich, wie ja auch in vielen seiner einfachen Salze das Cadmium, das, wie die Entstehung des Salzes $\mathrm{Cr}\left(\mathrm{C}_{2} \mathrm{O}_{4}\right)_{3} \mathrm{Cd} \cdot \mathrm{NH}_{4}+5 \mathrm{H}_{2} \mathrm{O}$ beweist, beständige Verbindungen zu bilden scheint.

Auch bei den doppelten Umsetzungen mit den Salzen der Metalle, wie Nickel, Kobalt oder Kupfer, tritt Zersetzung des komplexen Anions $\mathrm{II}\left(\mathrm{C}_{2} \mathrm{O}_{4}\right)_{3} \equiv$ ein. Hier ist jedoch der Reaktionsverlauf ein komplizierterer; denn es entstehen dabei die Alkalisalze eines anderen komplexen Anions ${ }^{\text {III }}\left(\mathrm{C}_{2} \mathrm{O}_{4}\right)_{2}$-, das, wie die Umsetzungsversuche mit rotem Chromoxalate (vergl. S. 463) zeigten, von grofser Beständigkeit zu sein scheint. Bei den Eisenalkalioxalaten tritt auch bei Umsetzung mit diesen Metallchloriden vollständige Zersetzung ein. Verbindungen des Anions $\mathrm{Fe}\left(\mathrm{C}_{2} \mathrm{O}_{4}\right)_{2}$ - können nicht entstehen, da dieses aufserordentlich labil ist. ${ }^{1}$ Dieses Verhalten scheint möglicherweise auf die saure Reaktion dieser Metallsalzlösungen zurückzuführen zu sein, doch lärst sich vorläufig hierüber nichts bestimmtes sagen.

Es bestätigen also diese Resultate im wesentlichen die am Schlusse der vorigen Mitteilung gezogenen Folgerungen, dals innerhalb des komplexen Anions schwach komplexsaurer Salze Zersetzung

\footnotetext{
1 Z. anorg. Chem. 11, 217.
} 
eintritt, sowie dasselbe mit Kationen von niedriger Zersetzungsspannung zusammentritt. Es sei hier nochmals hervorgehoben, dals diese Versuche, die einen weiteren Beitrag zu den Beziehungen zwischen Doppelsalzen und komplexen Salzen bringen sollten, vorläufig für diese Fragen nur einen qualitativen Wert zu besitzen beanspruchen.

Weitere Untersuchungen, die dieser Frage auch quantitativ näher kommen sollen, beschäftigen sich mit den Beziehungen der relativen Affinitätskonstanten komplexer Säuren zu den Affinitätskonstanten ihrer Komponenten. ${ }^{1}$ Es ist zu hoffen, dafs hierdurch ein tieferer Einblick in die Dissoziationsvorgänge der Mischsalze und ihre Abhängigkeit von den Affinitätsgrölsen gewonnen wird.

\section{Nachträge.}

Da hiermit die Versuchsreihe über die komplexen oxalsauren Salze, die sich über einen Zeitraum von fast 6 Jahren erstreckt, $\mathrm{zu}$ einem vorläufigen Abschluls gekommen ist, so seien hier noch einige Beobachtungen, die zur Ergänzung früherer Angaben dienen, nachgetragen.

1. Einige molybdänoxalsauren und vanadinoxalsauren Salze. Von Hermann Itzig. ${ }^{2}$

Während in der I. Mitteilung angegeben ist, dals die Natriummolybdänoxalate nicht krystallisieren, gelang es neuerdings beim Arbeiten mit gröfseren Mengen, dieselben in wohl charakterisierter Form zu isolieren.

Eine Lösung von $25 \mathrm{~g}$ neutralem Natriumoxalat wurde in der Siedehitze mit der auf 1 Mol. berechneten Menge Molybdänsäure versetzt. Die letztere löste sich vollständig auf und aus der auf dem Wasserbade fast bis zur Syrupkonsistenz eingeengten Lösung schied sich über Schwefelsäure alsbald ein leichtlösliches Salz in weifsen, in einander verwachsenen Nadeln ab. Dasselbe hatte die Zusammensetzung:

1 Dieselben sind von Herm Tн. A. Maass begonnen worden.

2 Ergänzung zur I. Mitteilung (Z. anorg. Chem. 4, 366). 


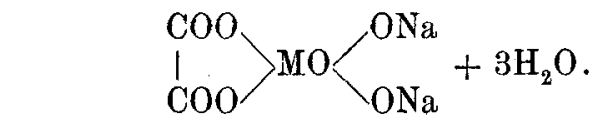

\begin{tabular}{|c|c|c|c|}
\hline \multicolumn{2}{|r|}{ Berechnet: } & \multicolumn{2}{|c|}{ Erhalten: } \\
\hline $\mathrm{Na}_{2} \mathrm{MO}_{4}$ & $62.05 \%$ & 62.55 & $62.73 \%$ \\
\hline $\mathrm{C}_{2} \mathrm{O}_{3}$ & 21.69 & 21.15 & 21.68 \\
\hline $3 \mathrm{H}_{2} \mathrm{O}$ & 16.26, & & \\
\hline
\end{tabular}

Eine Lösung von $25 \mathrm{~g}$ neutralem Natriumoxalat wurde in der Siedehitze mit Molybdänsäure vollständig abgesättigt. Die vom Überschusse der Molybdänsäure abfiltrierte Lösung ergab nach dem Einengen auf dem Wasserbade eine mikrokrystallinische Abscheidung eines weifsen Salzes der Zusammensetzung:

$$
\left.\right|_{\mathrm{COO}-\mathrm{MO}_{2}-\mathrm{ONa}} ^{\mathrm{COO}-\mathrm{MO}_{2}-\mathrm{ONa}}+6 \mathrm{H}_{2} \mathrm{O} .
$$

\begin{tabular}{lccc} 
& Berechnet: & \multicolumn{2}{c}{ Erhalten: } \\
$\mathrm{Na}_{2} \mathrm{M}_{2} \mathrm{O}_{7}$ & $66.04 \%$ & 66.32 & $66.33 \%$ \\
$\mathrm{C}_{2} \mathrm{O}_{3}$ & $13.50 \%$ & 13.70 & $13.69 \%$ \\
$6 \mathrm{H}_{2} \mathrm{O}$ & $20.46 "$ & &
\end{tabular}

$25 \mathrm{~g}$ neutrales Baryumoxalat wurde unter Zusatz der für $1 \mathrm{Mol}$. berechneten Menge Molybdänsäure in ungefähr $300 \mathrm{ccm}$ Wasser suspendiert und in einer Stöpselflasche bei gewöhnlicher Temperatur 3-4 Stunden in einer Schüttelmaschine stark durchgeschüttelt. Die suspendierte Masse hatte sich nach dieser Zeit vollständig in weifse Krystallnadeln verwandelt. Dieselben waren vollständig in Wasser von $50^{\circ}$, wenn auch etwas schwer, löslich; beim Stehen dieser Lösung jedoch, sowie beim Erwärmen auf höhere Temperatur trat Trübung infolge einer Zersetzung in Baryummolybdat und Baryumoxalat ein.

Die Analyse des Salzes führte zu der Formel:

$$
\left(\mathrm{MO}_{3} \mathrm{C}_{2} \mathrm{O}_{4}\right) \mathrm{Ba}+3{ }_{2} / \mathrm{H}_{2} \mathrm{O} \text {. }
$$

\begin{tabular}{|c|c|c|c|}
\hline & \multirow{2}{*}{$\begin{array}{c}\text { Berechnet: } \\
35.42 \%\end{array}$} & \multicolumn{2}{|c|}{ Erhalten: } \\
\hline $\mathrm{BaO}$ & & 35.61 & $35.71 \%$ \\
\hline $\mathrm{BaMO}_{4}$ & 68.75, & 68.70 & 68.85, \\
\hline $\mathrm{C}_{2} \mathrm{O}_{3}$ & 16.66 & 16.14 & $16.09 "$ \\
\hline $3^{1} /{ }_{2} \mathrm{H}_{2} \mathrm{O}$ & 14.58, & & \\
\hline
\end{tabular}

Durch doppelte Umsetzung einer mäfsig konzentrierten Lösung des vanadinoxalsauren Ammons: ${ }^{1}$

$$
\mathrm{NH}_{4} \mathrm{O}-\mathrm{VO}=\left(\mathrm{COO}-\mathrm{COO} \cdot \mathrm{NH}_{4}\right)_{2}+2 \mathrm{H}_{2} \mathrm{O}
$$

1 Z. anorg. Chem. 4, 369. 
mit äquivalenten Mengen von Baryumchlorid wurde ein in gelben, zu kugeligen Aggregaten vereinigten Nadeln krystallisierendes Baryumsalz erhalten. Es ist in kaltem Wasser schwer löslich.

$$
\mathrm{V}_{2} \mathrm{O}_{5}\left(\mathrm{C}_{2} \mathrm{O}_{4}\right)_{4} \mathrm{Ba}_{3}+15 \mathrm{H}_{2} \mathrm{O} \text {. }
$$

Berechnet:

$\begin{array}{ll}\mathrm{BaO} & 38.28 \% \\ \mathrm{C}_{2} \mathrm{O}_{5} & \mathbf{1 5 . 1 8} " \\ \mathrm{C}_{2} \mathrm{O}_{3} & 24.02 " \\ \mathrm{H}_{2} \mathrm{O} & 22.52 "\end{array}$

Erhalten:

$38.37 \quad 37.52 \%$

15.2715 .27 ,

24.1623 .72$,

2. Die äquivalente Leitfähigkeit der komplexen oxalsauren Salze.

\section{Von Ivan KoppeL. ${ }^{1}$}

In der II. Mitteilung sind die Leitfähigkeiten für die Verdünnungen von 250,500 und 1000 Liter u. s. w. verzeichnet. Da dieselben mit einem Apparate, der den Anforderungen der heutigen Forschung nicht entspricht, gemessen waren, so mögen hier die neuerdings ausgeführten genauen Bestimmungen für eine Reihe der Salze gemessen für die OsTWALD'schen Verdünnungen bei $25^{\circ}$ folgen:

\begin{tabular}{|c|c|c|c|c|c|c|c|}
\hline$v=$ & 32 & 64 & 128 & 256 & 512 & 1024 & $\begin{array}{c}\Delta= \\
\mu_{1024}-\mu_{32}\end{array}$ \\
\hline $\begin{array}{c}\text { Kaliumwolframoxalat: } \\
\mathrm{C}_{2} \mathrm{O}_{4}=\mathrm{WO}=(\mathrm{OK})_{2}+\mathrm{H}_{2} \mathrm{O}\end{array}$ & 62.0 & 66.4 & 70.3 & $\mathbf{7 3 . 7}$ & 79.0 & 84.0 & 22.0 \\
\hline $\begin{array}{l}\text { Kaliummolybdänoxalat sauer: } \\
\qquad \mathrm{C}_{2} \mathrm{O}_{4}=\mathrm{MO}<{ }_{\mathrm{OH}}^{\mathrm{OK}}+\mathrm{H}_{2} \mathrm{O}\end{array}$ & 71.2 & 85.5 & 109.4 & 131.7 & 156.8 & 178.5 & - \\
\hline $\begin{array}{l}\text { Kaliummolybdänoxalat neutral: } \\
\mathrm{C}_{2} \mathrm{O}_{4}=\mathrm{MO}=(\mathrm{OK})_{2}+\mathrm{H}_{2} \mathrm{O}\end{array}$ & 66.3 & 70.1 & 74.0 & 78.0 & 82.3 & 87.2 & 20.9 \\
\hline $\begin{array}{l}\text { Alkalivanadinoxalate: } \\
\mathrm{KO}-\mathrm{VO}=\left(\mathrm{C}_{2} \mathrm{O}_{4} \mathrm{~K}\right)_{2} \\
+3 \mathrm{H}_{2} \mathrm{O}\end{array}$ & 88.7 & 101.7 & 108.7 & 104.1 & 120.0 & 123.1 & 34.4 \\
\hline $\begin{aligned} \mathrm{NaO}- & \mathrm{VO}=\left(\mathrm{C}_{2} \mathrm{O}_{4} \mathrm{Na}\right)_{2} \\
& +7 \mathrm{H}_{2} \mathrm{O}\end{aligned}$ & 79.5 & 87.0 & 94.7 & 101.2 & 106.2 & 110.0 & 30.5 \\
\hline $\begin{aligned} \mathrm{NH}_{4} \mathrm{O}-\mathrm{VO}- & \left(\mathrm{C}_{2} \mathrm{O}_{4} \mathrm{NH}_{4}\right)_{2} \\
& +2 \mathrm{H}_{2} \mathrm{O}\end{aligned}$ & 85.7 & 93.6 & 100.3 & 106.5 & 110.0 & 113.8 & 28.1 \\
\hline Alkalichromoxalate: & & & & & & & \\
\hline $\mathrm{Cr} \equiv\left(\mathrm{C}_{2} \mathrm{O}_{4} \mathrm{~K}\right)_{8}+3 \mathrm{H}_{2} \mathrm{O}$ & 94.4 & 99.8 & 107.4 & 114.5 & 122.0 & 127.7 & 33.3 \\
\hline $\mathrm{Cr} \equiv\left(\mathrm{C}_{2} \mathrm{O}_{4} \mathrm{Na}\right)_{3}+4^{1} /{ }_{2} \mathrm{H}_{2} \mathrm{O}$ & 73.2 & 80.5 & 88.5 & 93.1 & 99.1 & 105.6 & 32.4 \\
\hline $\mathrm{Cr} \equiv\left(\mathrm{C}_{2} \mathrm{O}_{4} \mathrm{NH}_{4}\right)_{3}+3 \mathrm{H}_{2} \mathrm{O}$ & 93.2 & 101.3 & 112.5 & 115.9 & 123.0 & 129.0 & 35.8 \\
\hline $\mathrm{C}_{8} \mathrm{O}_{4}=\mathrm{Cr}-\mathrm{C}_{2} \mathrm{O}_{4} \mathrm{~K}+5 \mathrm{H}_{2} \mathrm{O}$ & 78.6 & 82.5 & 86.0 & 88.7 & 90.8 & 92.7 & 14.1 \\
\hline
\end{tabular}

1 Ergänzung zux II. Mitteilung (Z. anorg. Chem. 11, 225).

Z. anorg. Chem, XXI. 
$-18-$

\begin{tabular}{c|r|r|r|r|r|r|r}
\hline$v=$ & 32 & 64 & 128 & 256 & 512 & 1024 & $\begin{array}{c}\Delta= \\
\mu_{1024}-\mu_{32}\end{array}$ \\
\hline Alkalialuminiumoxalate: & & & & & & & \\
$\mathrm{Al}=\left(\mathrm{C}_{2} \mathrm{O}_{4} \mathrm{~K}\right)_{3}+2{ }^{1}{ }_{2} \mathrm{H}_{2} \mathrm{O}$ & 94.0 & 102.8 & 109.9 & 116.2 & 120.0 & 125.5 & 31.5 \\
$\mathrm{Al} \equiv\left(\mathrm{C}_{2} \mathrm{O}_{4} \mathrm{Na}\right)_{3}+5 \mathrm{H}_{2} \mathrm{O}$ & 73.9 & 80.0 & 89.4 & 96.0 & 100.0 & 104.2 & 30.3 \\
$\mathrm{Al}=\left(\mathrm{C}_{2} \mathrm{O}_{4} \mathrm{NH}_{4}\right)_{3}+2^{1}{ }_{2} \mathrm{H}_{2} \mathrm{O}$ & 95.5 & 102.4 & 108.8 & 114.8 & 118.7 & 124.8 & 29.3 \\
$\mathrm{C}_{2} \mathrm{O}_{4}=\mathrm{Al}-\mathrm{C}_{2} \mathrm{O}_{4} \mathrm{Na}+5 \mathrm{H}_{2} \mathrm{O}$ & 66.8 & 70.6 & 74.0 & 77.1 & 79.3 & 81.0 & 13.3 \\
\end{tabular}

Berlin N., Wissenschaftl.-chem. Laboratorium, 6. April 1899.

Bei der Redaktion eingegangen am 8. April 189. 